\title{
Clinicopathological profile of mediastinal masses in a tertiary care hospital of Eastern India
}

\author{
Saha B Kr ${ }^{1}$, Saha B. ${ }^{2}$, Sarkar D. ${ }^{3}$, Chakrabarti S. ${ }^{4}$, Bhattacharya S. ${ }^{5}$, Bandyopadhyay R. ${ }^{6}$ \\ ${ }^{1}$ Dr. Bikram Kr Saha, Assistant Professor, NBMC, Darjeeling, ${ }^{2}$ Dr. Biswajit Saha, Demonstrator, Murshidabad Medical \\ College, ${ }^{3}$ Dr. Debasis Sarkar, Assistant Professor, Malda MCH, ${ }^{4}$ Dr. Srabani Chakrabarti, Associate Professor Pathology, \\ CNMC, Kolkata, ${ }^{5}$ Dr. Sibasish Bhattacharya, Professor, Medical Oncology, MCH Kolkata, ${ }^{6}$ Dr. Ramtanu \\ Bandyopadhyay, Professor, Malda MCH, West Bengal, India.
}

Corresponding Author: Dr. Debasis Sarkar, Assistant Professor, Malda Medical College, Malda, West Bengal, India. E-mail: dr.debasis99bmc@yahoo.in

\begin{abstract}
Introduction: The Mediastinum is the central part of thorax where various organs are located which gives rise to various neoplastic and non- neoplastic lesions.Accurate diagnosis is important to formulate proper therapeutic strategy and predict prognosis. Studies are very few in this part of the country. Objective: To analyse presentation of patients with Meditastinal Mass and to classify according to the location of mass. Also, to document Malignant versus Non-malignant nature oflesions. Methods: Total 33 patients with mediastinal masses diagnosed by Imagingand Histopathological study were taken up consecutively in the study during one year of study. Results: of the 33 cases, 21 were male (63.6\%) and 12 were female (36.4\%), Male; female ratio being 1.8: 1. Age ranged from 14 years to 72 years maximum of 8 Patients $(24.2 \%)$ in the $12-20$ years of age group. $57 \%$ of the masses were malignant and $43 \%$ were benign. Most common lesion was Lymphoma in 10 cases (30.3\%) followed by8 cases of Thymic tumours (24.2\%). Metastatic Carcinoma was found in 6 patients (18.2\%) followed by 3 patients of Germ cell tumours. One case each of Neurofibroma, Neurolipoma and 6 are of different less common category. Compartment wise, 9 cases (27.3\%) were in Anterior Compartment, 1 (3\%) case in Post Compartment, 3 case in superior mediastinum, 6 case in Middle mediastinum. Maximum cases, 14(42.5\%) were occupying Multiple Compartments. Most common symptom was cough (72\%). Pleural and Pericardial effusions were common complication of malignant lesions contributing 7 out of $19(36 \%)$ and 3 out of 19(17\%) respectively. Conclusion: Mediastinal masses creates a diagnostic dilemma to the clinician. Uncommon cause of common symptoms like cough and common presentation of pleural or pericardial effusion may have Mediastinal mass behind the screen.
\end{abstract}

Key words: Mediastinal Mass, Lymphoma, Thymoma, Seminoma, Malignant effusions.

\section{Introduction}

Diseases of Mediastinum include primary tumors, metastatic tumors, cysts or acute and chronic infections. Primary mediastinal tumors are rare accounting for 3\% of tumours occurring within the chest [1]. Mediastinal mass is a term for mass (es) in the mediastinum.

The mediastinal space is narrow and cannot be expanded, thus a growth in this space will compress adjacent organs and cause a life-threatening emergency. Most mediastinal masses grow slowly, and thus patients often seek medical attention as the mass gets large enough, accompanied by signs of symptoms due to the compression of the mass to adjacent organs.

Manuscript received: $28^{\text {th }}$ October 2018

Reviewed: $7^{\text {th }}$ November 2018

Author Corrected: $16^{\text {th }}$ November 2018

Accepted for Publication: $22^{\text {nd }}$ November 2018
In comparison with reports from abroad, data regarding mediastinal mass in India is still scarce particularly in this part of the country. Mediastinal masses are relatively uncommon lesions that some times pose an interesting diagnostic and the rapeutic problem for the clinician [2]. The likelihood of malignancy is influenced primarily by the following three factors: mass location; patient age; and the presence or absence of symptoms.

Although more than two thirds of mediastinal tumours are benign, masses in the anterior compartment are more likely to be malignant. Age is an important predictor of malignancy as many of the lymphomas and germ cell tumours (GCTs) presents between the second and fourth decade of life. Last, symptomatic patients are more likely to have a malignancy. 
The most common symptoms at presentation are cough, chest pain, fevers/ chills and dyspnoea. Most symptoms can be categorized into the following two groups: localizing symptoms and systemic symptoms. Localizing symptoms are secondary to tumour invasion. Common localizing symptoms include respiratory compromise, dysphagia, paralysis of the limbs, diaphragm, and vocal cords, Horner syndrome, and superior vena cava syndrome. Systemic symptoms are typically due to the release of excess hormones, antibodies, or cytokines.

The initial workup of a suspected mediastinal mass involves obtaining posteroanterior and lateral chest radiographs. This can provide information pertaining to the size, anatomic location, density, and composition of the mass.

CT scanning is used to further characterize mediastinal masses and their relationship to surrounding structures as well as to identify cystic, vascular, and soft-tissue structure.

The role of MRI is primarily in ruling out or evaluating a neurogenic tumor. MRI is also valuable to evaluate the extent of vascular invasion or cardiac involvement.

Although nuclear scans and biochemical studies can be used to further characterize a lesion, tissue diagnosis is almost always required. If a mass is likely to be benign after initial workup, it can be removed surgically without biopsy. Otherwise, a diagnostic biopsy specimen can be obtained by transthoracic ortransbronchial needle aspiration, mediastinoscopy, anterior mediastinotomy, or video-assisted thoracic surgery, depending on the anatomic location and radiographic appearance of the lesion.

\section{Objectives}

1. To analyse age, sex and other socio-epidemiological factors in patients presenting with mediastinal mass.

2. To record presenting symptoms and physical findings of these patients.

3. To classify according to the location of the mass.

4. To record histopathological diagnosis and benign or malignant character of these masses.

\section{Methods}

a) Study Type: Hospital based Cross-sectional Observational study.

b) Sample Size: Total 33 patients with mediastinal masses were enrolled.

c) Sampling technique: Consecutive non probability technique used.

d) Inclusion Criteria: Patient diagnosed to have mediastinal mass clinically and readiologically. Both males and females above the age of 12 yrs attending OPD or admitted in Indoor of Medical College Hospital

e) Exclusion Criteria:a) Patients having primary oesophageal and cardiac tumoursb) Patients unwilling to join the study.

f) Study Procedure: The study commenced after obtaining permission from Institutional Ethical Committee and written informed consent from patients. Patients were selected with a provisional diagnosis of Mediastinal mass by CXR PA and Lat view, CT Scan of Chest. A detailed history and thorough clinical examination was done. Collection of laboratory data, imaging plates with reports and pathological reports were done.

Place of study: a) OPD and Indoor, Dept of Medical Oncology and Dept of Radiation Oncology Medical College Hospital, Kolkatab) OPD and Indoor, Dept of Medicine, Medical CollegeHospital, Kolkatac)OPD and Indoor, Dept of CTVS, Medical College Hospital, Kolkata.

Data analysis: All data gathered were tabulated on a master chart and analyzed using charts, diagrams and application of standard statistical techniques using latest SPSS software.

Statistical Methods- Categorical variables were expressed as Number of patients and percentage of patients. Continuous variables were expressed as Mean \pm Standard Deviation and compared across the groups using One- Way ANOVA test.

The statistical software SPSS version 20 has been used for the analysis. An alpha level of 5\% has been taken, i.e. if any $p$ value is less than 0.05 it has been considered as significant.

\section{Results and Analysis}

Out of 36 patients followed, 3 were excluded based on presence of various exclusion criteria (e.g. cardiac tumour-1 patients, oesophageal tumor 2 patient). Rest 33 patients of mediastinal mass, satisfying the inclusion criteria were analysed. Mediastinal masses has heterogeneous pathology. In this study the following diagnoses were obtained. 
Original Research Article

Table-1: Final diagnoses of Mediastinal masses.

\begin{tabular}{|c|c|c|}
\hline Final Diagnosis & No of Cases $(\mathrm{N})$ & Percent \\
\hline HL mixed cellularity & 1 & 3.0 \\
\hline Anaplastic large cell Lymphoma & 1 & 3.0 \\
\hline Large B cell Lymphoma & 3 & 3.0 \\
\hline Bronchial cyst & 1 & 3.0 \\
\hline Follicular Dendritic cell carcinoma & 1 & 3.0 \\
\hline HL NS Type & 2 & 6.1 \\
\hline Lymph node metastasis primary Lung & 5 & 15.1 \\
\hline Lymph node metastasis primary Breast & 1 & 3.0 \\
\hline Mature Cystic Teratoma & 1 & 3.0 \\
\hline Multi septate Thymic Cyst & 1 & 3.0 \\
\hline Neurofibroma & 1 & 3.0 \\
\hline Pericardial Cyst & 1 & 3.0 \\
\hline Retrosternal Goitre & 1 & 3.0 \\
\hline Seminoma & 2 & 6.1 \\
\hline Spindle cell sarcoma & 1 & 3.0 \\
\hline T Cell Lymphoblastic Leukaemia & 1 & 3.0 \\
\hline Inconclusive & 1 & 3.0 \\
\hline TB Lymphadenitis & 1 & 3.0 \\
\hline Thymic Carcinoma & 1 & 3.0 \\
\hline Thymic Lipoma & 1 & 3.0 \\
\hline Thymoma & 5 & 15.1 \\
\hline Total & 33 & 100.0 \\
\hline
\end{tabular}

Table-2: Age and sex distribution of patients:

Age distribution of the patients shows most number of cases are in younger age group 12- 20 yrs (8) followed by $21-30$ yrs and 31- 40 yrs (6) followed by 41- 60 yrs, 51- 60 yrs and 61-70 yrs, all have 4 . There is just one patient above the age of 70 yrs. Of the 33 patients 12 were female and 21 were male with male female ratio 1.8:1

\begin{tabular}{|c|c|c|}
\hline Age(Yrs) & Cases & Percent \\
\hline $12-20$ & 8 & 24.2 \\
\hline $21-30$ & 6 & 18.2 \\
\hline $31-40$ & 6 & 18.2 \\
\hline $41-50$ & 4 & 12.1 \\
\hline $51-60$ & 4 & 12.1 \\
\hline $61-70$ & 4 & 12.1 \\
\hline $71-80$ & 1 & 3.0 \\
\hline Total & $\mathbf{3 3}$ & $\mathbf{1 0 0 . 0}$ \\
\hline
\end{tabular}

\begin{tabular}{|c|c|c|}
\hline SEX & No of Cases & Percent \\
\hline Female & 12 & 36.4 \\
\hline Male & 21 & 63.6 \\
\hline Total & $\mathbf{3 3}$ & $\mathbf{1 0 0 . 0}$ \\
\hline
\end{tabular}




\section{Original Research Article}

\section{Table-3: Analysis of symptoms}

Out of the 33 patient only one (3\%) patientwas incidentally detected with mediastinal mass. Symptoms were classified as Local and Systemic.

a) Local: Among the local symptoms cough was the most commonest. 24 out of $33(72.7 \%)$ patients had complaint of cough. Dyspnea is the second common symptoms. 17 out of $33(51.5 \%)$ had dyspnea. Next symptoms in descending order are Chest pain (39\%), dysphagia (39\%), hoarseness of voice $(21.2 \%)$, bulging of chest wall $(12.1 \%)$.

\begin{tabular}{|c|c|c|}
\hline Symptoms & Cases & Frequency(\%) \\
\hline Cough & 22 & 72.7 \\
\hline Dyspnoea & 17 & 51.5 \\
\hline Chest pain & 13 & 39.4 \\
\hline Dysphagia & 13 & 39.4 \\
\hline Dysphonia & 7 & 21.2 \\
\hline Bulging of chest wall & 4 & 12.1 \\
\hline
\end{tabular}

b) Systemic Symptoms: Weight loss is the most common systemic symptom. 13 out of 33 cases (39.4\%) had history of weight loss. History of fever was present in 12 patients $(36.4 \%)$. Fatigue was complained by 10 patients $(30.3 \%)$. Complaint of excessive sweating was there in 6 patients $(18.2 \%)$.

\begin{tabular}{|c|c|c|}
\hline Symptoms & Cases & Frequency (\%) \\
\hline Weight loss & 13 & 39.4 \\
\hline Fever & 12 & 36.4 \\
\hline Fatigue & 10 & 30.3 \\
\hline Excessive sweating & 6 & 18.2 \\
\hline
\end{tabular}

\section{Table- 4: Complications}

Pleural effusion was present in $6(18.2 \%)$, pericardial effusion was present in $3(9.1 \%)$. There was features of Myasthenia Gravis in 3(9.1\%) patient. Superior Mediastinal Syndrome was present in 4 (12.2\%) patients and Horner Syndrome was present in $1(3 \%)$ patient.

\begin{tabular}{|c|c|c|}
\hline Complications & Cases & Frequency $(\%)$ \\
\hline Pleural effusion & 6 & 18.2 \\
\hline Pericardial effusion & 3 & 9.1 \\
\hline Sup Mediastinal syndrome & 4 & 12.2 \\
\hline Horner syndrome & 1 & 3 \\
\hline Myasthenia Gravis & 3 & 9.1 \\
\hline
\end{tabular}

\section{Table-5: Location of masses in CT scan}

Anterior and anterio-superior compartment of the mediastinum is most common location of mass followed by middle and posterior compartment.

\begin{tabular}{|c|c|c|}
\hline Compartment & Cases & Percent \\
\hline Anterior & 9 & 27.3 \\
\hline Posterior & 1 & 3 \\
\hline Superior & 3 & 9.1 \\
\hline Middle & 6 & 18.2 \\
\hline Mixed & 14 & 42.5 \\
\hline Total & $\mathbf{3 3}$ & $\mathbf{1 0 0 . 0}$ \\
\hline
\end{tabular}


Table-6: Pathological Character

$19(57.6 \%)$ of the total 33 cases were malignant lesion as revealedby radiological features, FNAC and Biopsy. Rest $14(42.4 \%)$ were benign in nature.

\begin{tabular}{|c|c|c|}
\hline Group & Cases & Percent \\
\hline Benign & 14 & 42.4 \\
\hline Malignant & 19 & 57.6 \\
\hline Total & $\mathbf{3 3}$ & $\mathbf{1 0 0 . 0}$ \\
\hline
\end{tabular}

Table-7: Types of Mediastinal Masses

Mediastinal masses are diagnosed clinically radiologically and by histology. 10 (30.3\%) patients were diagnosed as having lymphoid malignancy. $8(24.2 \%)$ had tumours of thymic origin. $3(9.1 \%)$ had Germ cell tumours and $6(18.2 \%)$ had metastatic carcinoma.

\begin{tabular}{|c|c|c|}
\hline Types of mediastinal masses & Cases & Percent \\
\hline Thymic tumours & 8 & 24.2 \\
\hline Lymphoma & 10 & 30.3 \\
\hline Germ cell tumours & 3 & 9.1 \\
\hline Metastasis & 6 & 18.2 \\
\hline Others & 6 & 18.2 \\
\hline Total & $\mathbf{3 3}$ & $\mathbf{1 0 0 . 0}$ \\
\hline
\end{tabular}

Thymic Tumors: Among thymic tumours 5 were thymoma (62\%), 1 thymic cyst (13\%),1 thymic lipoma (13\%) and 1 thymic carcinoma $(13 \%)$.

Myasthenia in Thymic Tomours: Myasthenia Gravis was diagnosed in 3 patients (37.5\%) among thymictumours

Lymphomas: Among 10 patients (30.3\%) of lymphomas 6 were NHL (60\%) and 3 were HL(30\%) and 1 was indeterminate $(10 \%)$.

Germ cell tomours: Among 3 cases of germ cell tumours 2 were seminomas and 1 mature cystic teratoma.

Metastatic carcinoma: Among Metastatic Carcinoma, Lung carcinoma was found to bein 4 patients and Breast Carcinoma was found to be in 1 case.

Others: Beside these there is 1 Retrosternal goiter, 1 TB lymphadenitis, 1 neurofibroma, and 1 spindle cell sarcoma was found

FNAC: Fine needle aspiration cytology or core biopsy been done in 21 cases $(63 \%)$ cases.

\section{Discussion}

Most common cause found in this study is lymphoidmalignancy.There were 10 lymphoma cases $(30.3 \%)$ of which 4 were having isolated mediastinal lymphadenopathy. Among these 10 patients, 6 were NHL and 3 were HL and 1 unspecified. Among6 NHL patients Diffuse large B Cell Lymphoma was commonest with 3 cases, $\mathrm{T}$ cell lymphoblastic lymphoma/ leukemia was present in 2 patients. Second most common type of tumours found were thymic tumours, 8 cases $(24.2 \%)$. Most of the thymic tumour were benign with stage I and stage II disease. There was one thymic cyst and one thymic carcinoma. Metastatic carcinoma of lymphnode was detected in 6patients (18.2\%). Primary was mostly lung carcinoma (5 patients). Among 3 cases $(9.1 \%)$ of germ cell tumour two were seminomas and one was mature cystic teratoma proven by histopathology. There was one case of neurofibroma with multiple lesion in mediastinum and lung, one case of mediastinal lymhadenopathy along with cervical lymphadenopathy. Two cystic 


\section{Original Research Article}

lesions were found one was pericardial cyst and another was bronchogenic cyst.There was one case of retrosternal goiter proved by FNAC and thyroid scan and one case of spindle cell sarcoma. Most of the masses were malignant in character. $19(57.6 \%)$ of total 33 cases were malignant and rest $42 \%$ were benign, this is some what similar to study of vaziri et al [3] However, benign lesions were more common in study conducted by Adegboye et al., (57\%) [4] and Davis et al., $(58 \%)[5]$.

This would reflect the increasing incidence of malignancy over the years. Mediastinal masses are most common younger age group which is in comparable to studies done by Dubashi et al [6]. Most cases were found in the age group of twelve to twenty. Metastasis was common in older age group. 5 out of 6 patient was above age 50. Thymic tumours occurred most commonly in the thirty to forty years age group. 4 out of $8(50 \%)$ was in this group. Among the lung carcinoma with mediastinal metastasis cases 4 out of 5 were smoker.

However there is no significant association between smoking with other malignant masses. The median time of duration of symptoms was 19 weeks for benign lesions and 7 weeks for malignant lesions, significantly lesser in malignant group. $32(97 \%)$ patient with mediastinal mass was symptomatic, while only in $1(3 \%)$ the mass was detected incidentally while doing a $\mathrm{x}$ ray for pre anesthetic assessment for a surgery, which is in comparison with study done by Singh et al., (94.7\%)[7] and Dubashi et al., (97\%).

Higher incidences of asymptomatic cases were found in study by Vaziri et al., (12\%), Adegboye et al., (22.9\%)and Davis et al., (38\%). This observation may be due to the fact that many of our patients visit the hospital for their symptoms rather than for routine evaluation. As majority of our cases were malignant, this may reflect the fact that malignant tumours are more symptomatic than benign tumours.

Location of mass is diagnosed by CT scan imaging of thorax. Thymoma (40\%) was the commonest tumour in the anterior mediastinum, followed by lymphoma (33.3\%). Middle mediastinal involvement was seen in $11.43 \%$ cases, which is comparable with other studies $[5,9]$. However, the incidence of tumour in the posterior mediastinum $(8.57 \%)$ was much less in comparison with other studies by Adegboye et al [4] (22.9\%) and Davis et al[5] (26\%). This could be due to the lack of neurogenic tumours in our study. Multiple compartments involvement by malignant lesions is more commonly encountered due to local spread of tumour. A Retrospective Study conducted by Dubashi B et al[6] showed Primary mediastinal tumors were seen common in males with mean age of $37.48 \pm 17.04$ years. About $97 \%$ of patients were symptomatic at presentation.

Superior venacaval obstruction (SVCO) was seen in $28 \%$ of the patients. About $50 \%$ of the patients were diagnosed by a fine-needle aspiration or True-cut biopsy, while $28 \%$ of the patients required thoracotomy for a diagnosis. Majority of the tumors had anterior mediastinal presentation. Pleural effusion was seen in $20 \%$ of the patients, but diagnosis was obtained in only $1 \%$. In adults, thymoma (39\%), lymphoma (30\%) and germ cell tumor $(15 \%)$ were the common tumors.

A study done by Jitendra G Nasit et al [8] concluded that the rates of nonsurgical tumors such as lymphoma are higher and the rates of traditionally surgical diseases such as thymomas are lower. Prompt and correct diagnosis of anterior mediastinal masses is the key process in therapeutic decision. The precise nature of anterior mediastinal masses cannot be determined without histology examination of the tissue.

Chandra P Shrivastava et al [9] showed in their study Ages ranged from 6 months to 62 years, with peak incidence inthe third and fourth decade of life (56\%). The male to female ratio was 1.9:1. Theanterosuperior mediastinum was involved in 76 patients $(72 \%)$, middle mediastinum in $13(12 \%)$, and posterior mediastinum in $17(16 \%)$. Myasthenia gravis was present in $27 \%$ of cases. Histopathologically,41 (39\%) patients had thymic pathology, 31 (29\%) had lymphoma, 14 (13\%) hadgerm cell tumors, 12 (11\%) had neurofibroma, 4 (4\%) had ganglioneuroma, 2 (2\%) had bronchogenic cyst, and 1 each had thymic cyst and mesothelioma.

In a study done by Blegvard S et al [10] of 129 operated mediastnal tumours, Intrathoracic goitre, neurogenic lesions and thymomas comprised $62 \%$ of all the tumours. Fifty lesions (39\%) were found at routine radiographic examination and were asymptomatic. Eight of these 50 lesions were malignant. Chest pain, fatigue, weight loss and fever were significantly more common in malignant than in benign disease.

Analysis of 139 cases of mediastinal lesions by FNAC and/or Biopsy by R Dixit et al [11] showed 93 cases were neoplastic in nature $(67 \%), 32$ were nonneoplastic $(23 \%)$, and 14 remained inconclusive (10\%). Among 


\section{Original Research Article}

neoplastic mediastinal lesions, metastatic carcinoma $(37.4 \%)$ was the most common neoplastic lesion, followed by non-Hodgkin's lymphoma (12.2\%), Hodgkin's lymphoma (7.1\%), thymic lesions (3.5\%), etc. Among nonneoplastic conditions, tuberculosis was the most common lesion (20.1\%).

In a small review of 16 operated cases by Malatani TS [12] showed Anterior mediastinal tumours included retrosternal goitre (2), benign cystic teratoma (1), benign thymoma (1), malignant thymoma, spindle cell type (1) and Hodgkin's lymphoma, nodular sclerosing type (1). Mid-mediastinal tumours included bronchial cyst (1), mediastinal granuloma (1), and pulmonary arterio-venous fistula (1). Neurilemmoma (2), neuroblastoma (1), ganglioneuroma (1), Askin tumour (1), neurofibroma (1) and benign histiocytoma (1) constituted the tumours of the posterior mediastinum.

Abebe Bekele et al [13] studied patterns of mediastinal tumours operated in a Soudi Arabia teaching Hospital and reported a different types of presentations. He showed that in a six years review of 73 patients who were operated, $49(67.1 \%)$ were males, the and male to female ratio being 2.04:1. The mean age of patients was $35.9+/-10.5$ years (range 14 to 74$)$. Forty-five $(61.6 \%$ ) had lesions of the anterior mediastinum, 23 (31.5\%) in the posterior mediastinum and $5(6.8 \%)$ in the middle. The commonest anterior mediastinal tumors were thymic origin (24/45), and thymic lesions were found more common in females (17:7 ratio). From the 23 patients with posterior mediastinal tumors, 18 had benign neurogenic tumors (4 of which were dumbbell tumors). Chest pain and shortness of breath (dyspnea) were the two most common symptoms in 31 (42.4\%) of the patients. Twenty three patients $(31.5 \%)$ were asymptomatic, and all had benign lesions. None of the malignant lesions were asymptomatic. The rate of malignancy in this study was 24 (32.8\%), of which 19 $(79.1 \%)$ were in the anterior compartment.

\section{Conclusions}

1. In our study, the most common tumour in mediastinum was lymphoid malignancy. Thymoma is the second most common tumours. There are other diverse group of masses and cysts in mediastinum including germ cell tumours, cysts, metastatic carcinoma, sarcoma and nerve sheath tumours.

2. In our study lymphomas were distributed among younger age groups and thymomas and germ cell tumoursand cysts in the middle age group and metastatic tumours in the older age group.
3. Most common presentation was cough followed by dyspnea, chest pain, fever, malaise, dysphagia, weight loss. Constitutional symptoms were more common in lymphoid malignancy and metastasis.

4. Physical sign was unremarkable in many cases. Most common findings were pallor, cervical lymphadenopathy, dull mediastinal percussion note

5. Mostcommon complication is pleural effusionfollowed by pericardial effusion and horner's syndrome.

Limitations- To make sample size significant we need longer duration of study or conduct a retrospective study from the hospital register, but extracting datas from remote past was difficult in our setting. In case of lymphoid malignancy there is no cut off value for size of the lymphnode to call it a mass.

It is based on the observation of radiologist. In case of thymic tumours there is no distinct differentiation between size of normal thymus, thymic hyperplasia and thymic tumours. In some cases biopsy could not be done before surgery. Video assisted thoracoscopy and Video assisted mediastniscopy facilities are not always available in our institute.

Though this study is not a new in the field, there is no review of present occurrences of mediastinal masses and their nature particularly in this part of the country. In view of increasing occurrence of malignant disorders, we planned for this study to see if there is any changing pattern of types of diseases.

\section{Contribution by authors}

1. Bikram Krsaha and Biswajit Saha: Concept Design, conducting the study and writing the manuscript.

2. D. Srakar: Conducting study and writing Manuscript.

3. S. Chakabarti: Histopathological studies.

4. S. Bhattacharya and R Bandyopadhyay: Guiding the study procedure and preparing the manuscriptsuitable for publication.

Funding: Nil, Conflict of interest: None

Permission of IRB: Yes

\section{References}

1. Aroor AR, Prakasha S R, Seshadri S, et al. A study of clinical characteristics of mediastinal mass. J Clin Diagn Res. 2014 Feb;8(2):77-80. doi: 10.7860/JCDR/ 2014/7622.4013. Epub 2014 Feb 3. 


\section{Original Research Article}

2. Duwe BV, Sterman DH, Musani AI. Tumors of the mediastinum. Chest. 2005 Oct; 128(4):2893-909.DOI: 10. 1378 / chest.128.4.2893

3. Vaziri M, Pazooki A, Zahedi-Shoolami L. Mediastinal masses: Review of 105 cases. Acta Med Iranica 2009; 47(4):297-300.

4. Adegboye VO, Ogunseyinde AO, Obajimi MO, et al. Presentation of primary mediastinal masses in Ibadan. East Afr Med J. 2003 Sep;80(9):484-7.

5. Davis RD Jr, Oldham HN Jr, Sabiston DC Jr. Primary cysts and neoplasms of the mediastinum: recent changes in clinical presentation, methods of diagnosis, management, and results. Ann Thorac Surg. 1987 Sep; 44 (3):229-37.

6. Dubashi B, Cyriac S, Tenali SG. Clinicopathological analysis and outcome of primary mediastinal malignancies - A report of 91 cases from a single institute. Ann Thorac Med. 2009 Jul; 4(3):140-2. doi: 10. 4103/1817-1737.53354.

7. Singh G, Amin Z, Wuryantoro, Wulani V, Shatri H. Profile and factors associated with mortality in mediastinal mass during hospitalization at Cipto Mangunkusumo Hospital, Jakarta. Indonesian Journal of Internal Medicine.2013; 45:3-10.
8. Jitendr G Nasit, Maulin Patel, Biren Parikh, Manoj Shah, KajalDavara. Anterior mediastinal masses: A study of 50 Cases by fine needle aspiration cytology and core needle biopsy as a diagnostic procedure. South Asian Journal of Cancer.2013 Jan-March; 2(1):7-13.

9. Shrivastava CP, Devgarha S, Ahlawat V. Mediastinal tumors:a clinicopathological analysis. Asian Cardiovasc Thorac Ann. 2006 Apr; 14 (2):102-4. DOI:10. 1177/ 021849230601400204

10. Blegvad S, Lippert H, Simper LB, et al. Mediastinal tumours. A report of 129 cases. Scand J Thorac Cardiovasc Surg. 1990;24(1):39-42.

11. Ramakant Dixit, Narender Singh Shah, Mukesh Goyal, Chetan B Patil, Mukesh Panjabi, Rakesh C Gupta, Neeraj Gupta, Sabarigiri Vasan Harish. Diagnostic evaluation of mediastinal lesions: Analysis of 144 cases. Lung India $2017 ; 34(4): 341-348$. DOI: 10.4103/lungindia.lungindia_311_16.

12. Malatani TS. Primary mediastinal tumours in the southern region of Saudi Arabia. Indian J Chest Dis Allied Sci. 1996 Oct-Dec;38(4):235-9.

13. Bekele A, Ali A, Gulilat D, Kassa S, Nega B. Patterns of mediastinal tumors operated at the Tikur Anbessa Hospital, Addis Ababa, Ethiopia over a six years period. Ethiopian Medical Journal 01 Apr 2013; $51(2): 143-152$.

\section{How to cite this article?}

Saha B K, Saha B, Sarkar D, Chakrabarti S, Bhattacharya S, Bandyopadhyay R. Clinicopathological profile of mediastinal masses in a tertiary care hospital of Eastern India. Int J Med Res Rev 2018;6(08):427-434. doi:10.17511/ijmrr.2018.i08.06. 